\title{
UNE ALTERNATIVE AU "TOUT NARRATIF": LES GRADIENTS DE NARRATIVITÉ
}

\author{
Jean-Michel Adam ${ }^{1}$
}

Les systèmes symboliques sont des artefacts. Leurs traits syntaxiques et sémantiques ne sont pas dictés par le champ, mais résultent de décisions que nous prenons concernant la façon dont ce champ doit être organisé. Les systèmes que nous construisons déterminent les similarités et les différences que nous reconnaissons, les niveaux de précision que nous pouvons produire, les degrés de détermination que nous pouvons atteindre. (...)

En construisant un système, nous ne partons jamais de zéro. Nous commençons inévitablement avec certaines conceptions des objets du domaine et avec certaines convictions qui les concernent. Cela guide nos constructions. En construisant un système verbal, notre but est d'organiser un domaine d'une façon telle que nous puissions formuler de plus en plus d'énoncés intéressants et précis à son sujet ${ }^{2}$.

L'hypothèse principale du livre que j'ai consacré aux Textes : types et prototypes ${ }^{3}$ est celle de gradients de typicalité. Dans cette

1 Professeur de linguistique française à l'Université de Lausanne. Centre de Recherches en Linguistique Textuelle et Analyse des Discours.

2 N. GoODMAN et C. Z. ELGIN, Reconceptions en philosophie, dans d'autres arts et dans d'autres sciences, Paris, PUF, 1994, p. 11-12.

3 J.-M. ADAM, Les textes: types et prototypes, Paris, Nathan, coll. FAC, 1992.

Recherches en communication, $\mathrm{n}^{\circ} 7,(1997)$. 
perspective, tout texte est plus ou moins narratif, plus ou moins descriptif, plus ou moins argumentatif, plus ou moins explicatif ou encore dialogal. Comme le souligne la réflexion épistémologique citée en exergue, la façon dont je choisis d'organiser, en le théorisant, le domaine est un point de vue particulier sur les textes/discours, un point de vue déterminé par une finalité descriptive: "formuler de plus en plus d'énoncés intéressants et précis" à propos des textes narratifs.

\section{Un point de vue linguistique sur les textes/discours}

Comme le dit très clairement le second paragraphe du texte de Nelson Goodman et de Catherine Z. Elgin cité en exergue, "nous ne partons jamais de zéro", nous héritons d'une certaine conception -pour nous de la narratologie- par rapport à laquelle un travail de "reconception" peut être nécessaire. J'ai beau avoir écrit trois essais sur le sujet, le récit et la narratologie ne sont, pour moi, ni un objet donné ni une discipline autonome.

\subsection{Remarques historiques et épistémologiques}

Si le récit a occupé le devant de la scène et si la narratologie est une discipline dont les développements modernes ont été fulgurants de la fin des années 1960 aux années 1980 -quoiqu'en dise un Jean Molino qui considère que "l'analyse des récits en est encore à l'âge des maladies infantiles, où l'on se borne à répéter avec un vocabulaire ambitieux des données simplistes"1-, il me semble que c'est peut-être dû à l'importance des formes narratives dans notre culture, mais c'est surtout un fait de conjoncture, lié aux développements du structuralisme linguistique et, en particulier, au succès, dans les années 1960, du texte de Propp sur la morphologie du conte. Ce succès tient à l'influence de ses plus prestigieux commentateurs : Claude Lévi-

1 J. Molino, "Thèses sur le langage, le discours, la littérature et le symbolique", Zeitschrift für französische Sprache und Literatur, Stuttgart, Franz Steiner Verlag, Band C 1990, p. 166. 
Strauss et Algirdas Julien Greimas d'abord, Claude Bremond et Paul Larivaille par la suite ${ }^{1}$.

Selon moi, à la fin des années soixante, le succès de l'analyse structurale des récits (avec le numéro 8 de Communications) tient à l'absence de linguistique du texte et du discours et aux besoins d'une critique littéraire universitaire en crise. Les travaux de Genette, de Barthes et du premier Todorov, ont offert le pendant, pour la prose, des analyses de la poésie issues des propositions de Roman Jakobson et de Nicolas Ruwet. Tous ces travaux ont occupé le terrain de la textualité laissé libre par une linguistique qui s'était fixé la phrase pour ultime limite.

Je crois qu'il faut insister sur le fait que Genette et Todorov ont plus été d'admirables lecteurs de textes romanesques que des théoriciens du récit. C'est par leurs descriptions qu'ils ont eu l'influence que l'on sait.

La théorie narrative d'un Umberto Eco a pris progressivement sens dans le cadre plus large de ce qu'il nomme lui-même, au début de Lector in fabula, une pragmatique du texte. Ceci contraste très sensiblement avec le véritable effondrement des narratologies de Barthes et de Todorov. L'un comme l'autre ont cessé de faire de la narratologie. Sans cadre théorique capable de l'intégrer, la narratologie structurale se dilue d'elle-même.

Les efforts récents de Gérard Genette (je pense au chapitre de Fiction et diction intitulé "Récit fictionnel, récit factuel") pour interroger la pertinence générale de ses propositions me paraissent significatifs de ce besoin de la narratologie de s'ouvrir: "Si les mots ont un sens (et même s'ils en ont plusieurs), la narratologie (...) devrait s'occuper de toutes les sortes de récits, fictionnels ou non"2. Comme le note $\mathrm{G}$. Genette, le travail d'un Barthes, malgré un très timide détour par l'écriture narrative de l'histoire, est resté strictement littéraire. Genette parle très justement d'un "privilège implicite qui

1 Je passe sur le fait éditorial qui isole la Morphologie des Racines historiques. Il est particulièrement significatif que ce soit sur une æuvre amputée de Propp que la narratologie structuraliste se fonde... Pour un historique plus complet sur la question de l'héritage formaliste, je renvoie au chapitre 2 de Le récit, (Paris, PUF, coll. Que sais-je?, ${ }^{\circ} 2149,1984$ ).

2 G. GENETTE, "Récit fictionnel, récit factuel", in Fiction et diction, Paris, Éd. du Seuil, 1991, p. 65. 
hypostasie le récit fictionnel en récit par excellence, ou en modèle de tout récit"'.

Disant cela, je bats évidemment ma propre coulpe, ayant jadis intitulé Discours du récit une étude manifestement confinée au récit de fiction et récidivé naguère dans Nouveaux discours $d u$ récit, malgré une protestation de principe contre cette pratique trop unilatérale de ce qu'il faut bien appeler une narratologie restreinte ${ }^{2}$.

Il me semble qu'un autre exemple confirme mon analyse : la "sémiotique narrative" de l'École de Paris, la plus fidèle héritière du projet structuraliste, n'existe précisément que de ne plus être "narrative". D'être certes une sémiotique, mais pas du tout une narratologie... Par un intéressant paradoxe, la «narrativité généralisée» qui caractérise les travaux de Greimas et de ses épigones a dilué le projet narratologique dans un projet sémiotique beaucoup plus vaste. Il ne me semble pas du tout surprenant de voir un Jean-Claude Coquet ou un Jacques Fontanille mettre l'énonciation en avant dans leur projet sémiotique. Cette narrativité «vidée -selon Greimas lui-même- de son contenu conceptuel» ${ }^{3}$ devient un «principe organisateur de tout discours» ${ }^{4}$ qu'il ne sert plus à rien de qualifier encore de "narratif".

Que la production du sens implique des schèmes dynamiques, des suites d'opérations et des séries logiques, des algorithmes de transformations, des parcours orientés, bref, que la signification s'organise syntaxiquement, on ne saurait le contester, et ce n'est pas le moindre mérite de la sémiotique que de l'avoir mis en évidence; mais cette syntaxe doit-elle encore être qualifiée de "narrative", dès lors qu'elle ne régit plus des processus figuratifs, ou impliquant au moins une représentation temporelle? Le muthos n'est pas le logos 5 .

1 Ibid.

2 lbid., p. 66.

3 A. GreImas, Du sens, vol. II, Paris, Éd. du Seuil, 1983, p. 18.

4 A. Greimas et J. Courtès, Sémiotique, dictionnaire raisonné de la théorie du langage, Paris, Hachette, 1979, p. 249.

5 M. MATHIEU-COLAs, "Frontières de la narratologie", Poétique $n^{\circ} 65$, Paris, Éd. du Seuil, 1986, p. 106-107. 


\subsection{Une "reconception" de la narratologie}

Pour moi, les discours en général et les discours médiatiques en particulier sont des pratiques discursives qu'il faut étudier dans leur dimension communicationnelle-discursive, d'une part, et dans leur dimension textuelle, plus purement linguistique, d'autre part. C'est ce qu'illustrent les deux parties du schéma général de la textualité (partie inférieure) et de la discursivité (partie supérieure), parties aussi inséparables, pour reprendre l'analogie saussurienne, que le recto et le verso d'une feuille de papier.

Dans cette perspective, tel organe de presse écrite (Le Soir, Le Monde, Libération, Le Nouveau Quotidien) est une institution et le producteur individuel ou collectif de tel article fait partie d'une formation discursive. C'est dire qu'il est pris dans l'interdiscours de la presse en général et de tel organe de presse -régionale ou nationalede France ou de Belgique ou de Suisse francophones. Son discours est interdiscursivement et intertextuellement réglé, conditionné par les genres et sous-genres de la presse écrite. C'est à ce niveau de généricité discursive que la formation discursive de la presse nous fournit des catégorisations qui ont une histoire : "billet d'humeur", "fait divers", "petites annonces", "éditorial" ou "reportage". C'est à ce niveau également que l'on peut situer les grilles des "familles événementielles"1 qui préorganisent l'information, sa distribution comme sa reconnaissance.

1 M. Mounlaud et J.-Fr. TÉTu, Le journal quotidien, Lyon, Presses universitaires de Lyon, 1989. 


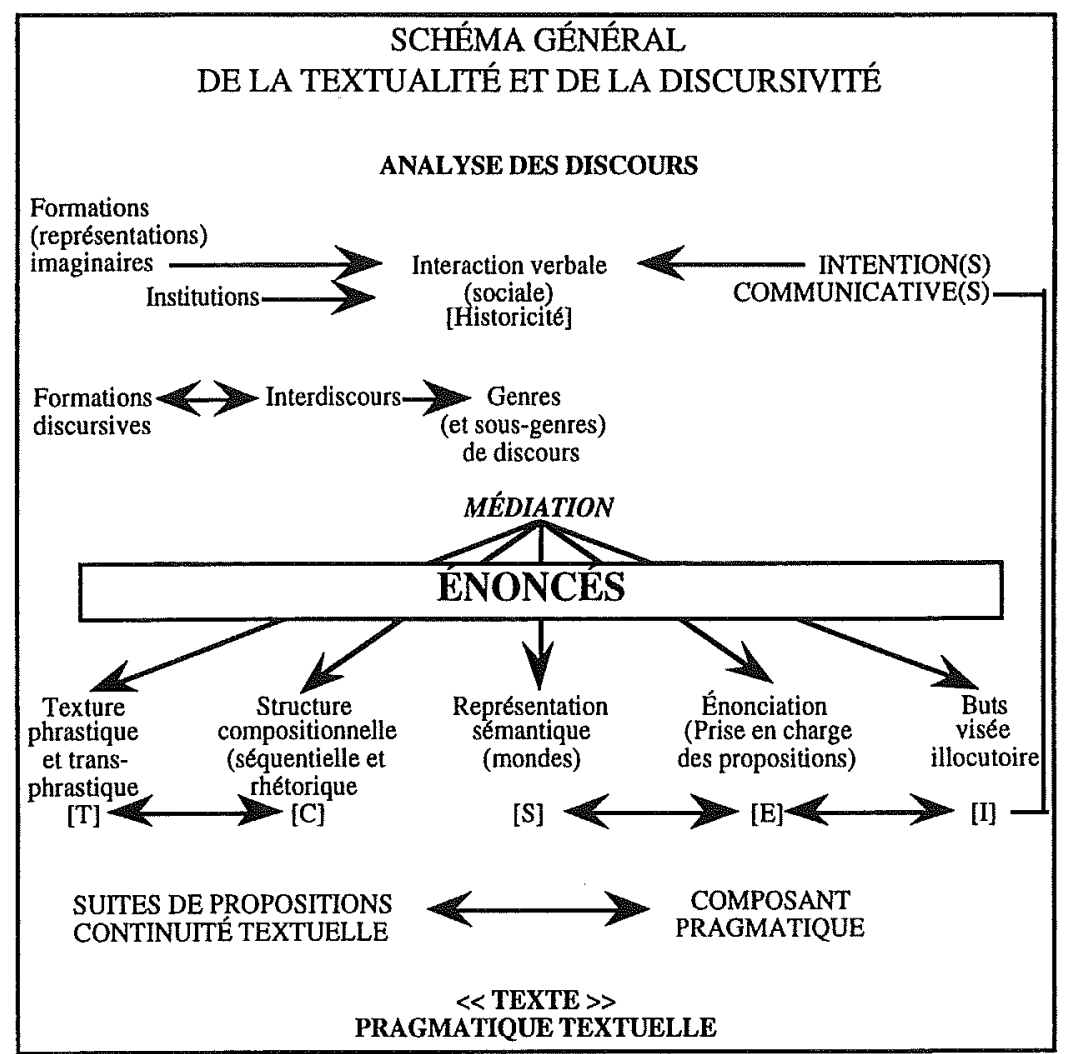

Je ne développe pas cette approche du recto discursif des énoncés médiatiques. Sous son verso textuel, un énoncé médiatique est une produit hétérogène et complexe que je théorise comme un système d'interrelations entre différents plans de structuration.

Trois plans de l'organisation pragmatique peuvent être distingués : la visée illocutoire [I], la prise en charge énonciative des propositions [E] et la représentation construite ou "monde" du texte [S]. Deux plans d'organisation assurent l'articulation des propositions : la grammaire de phrase et la grammaire de texte sont responsables de ce qu'on peut appeler la texture phrastique et transphrastique [T]. Il faut ajouter à ce plan d'organisation de toutes les formes de mise en texte un autre plan, celui de la structure compositionnelle des textes [C] qui va nous ramener à la question des catégorisations des textes et nous 
permettre de croiser catégorisations génériques et catégorisations plus proprement linguistiques et textuelles ${ }^{1}$.

Ces cinq plans d'organisation complémentaires, liés probablement à divers systèmes de connaissances des sujets, coopèrent et s'enchaînent très étroitement lors des processus de production et d'interprétation.

\section{TEXTURE [plan T d'organisation]}

- TEXTURE PHRASTIQUE : Domaines classiques de la linguistique

- TEXTURE TRANSPHRASTIQUE : allant du phonème au syntagme.

Liages transphrastiques allant de l'anaphore et de la progression thématique aux faits de connexion en général (organisateurs et connecteurs, structure de la période).

Segmentation (recouvrant tous les phénomènes de ponctuation liés à la matérialité de la mise en texte écrite).

Une théorie des plans d'organisation apparaît comme nécessaire dans la mesure où les solidarités syntaxiques (structurales et locales) n'ont qu'une portée discursive très limitée : "Une fois que l'on est sorti du domaine de localité dans lequel ces connexions peuvent fonctionner, d'autres systèmes de connexion interviennent. Ces systèmes ne reposent pas sur des critères structuraux, ils mettent en jeu des marques ou, plus exactement, des instructions relationnelles capables d'exercer leur pouvoir à longue distance"2. Le besoin d'une définition des plans d'organisation résulte de l'objet transphrastique de la linguistique du texte. En effet, les connexions proprement textuelles sont, d'une part, capables de fonctionner à longue distance et, d'autre part, à la différence des connexions phrastiques, elles n'entrent pas dans des schémas aussi contraints que les schémas syntaxiques. Ceci explique que le texte soit une "entité structuralement ouverte"3, nécessitant un corps de concepts descriptifs propre: "La constatation que les domaines textuels et morphosyntaxiques sont -dans une large mesure- indépendants, que la

1 Exposées dans J.-M. ADAM, Les textes..., op.cit.

2 M. Charolles, "Les plans d'organisation du discours et leurs interactions", in Parcours linguistiques de discours spécialisés, Berne, Peter Lang, 1993, p. 305.

3 Ibid., p. 311. 
cohérence du texte n'est pas la résultante de faits de grammaticalité, conduit aussi à s'interroger sur la pertinence des catégories linguistiques habituellement reconnues ${ }^{1}$.

Les plans d'organisation sont constitués d'unités qui ne s'intègrent pas les unes dans les autres pour former, par emboîtement hiérarchique, des unités de rang supérieur. On a affaire plutôt à un développement en parallèle de systèmes de solidarité, et les marques appartenant aux divers plans ou modules sont amenées soit à se corroborer et à se renforcer, soit, au contraire, à s'inhiber et à se contrecarrer. L'étude de ces relations et leur modélisation est la tâche principale de la linguistique textuelle, telle du moins que nous la concevons. En raison des interactions entre les plans d'organisation, le cadre de cette théorisation ne peut être que celui des modèles intégrant la complexité. Avec M. Charolles, nous parlerons de modèles «de type interactif et massivement parallèle dans lesquels on jouera sur des constellations d'indices pondérés s'inhibant ou se renforçant $»^{2}$.

Linguistique modulaire, d'Henning $\mathrm{N} \varnothing \mathrm{ke}^{3}$, est certainement un des meilleurs exemples de cette tendance actuelle de la réflexion linguistique. Sans nous prononcer ici sur les modules retenus par les uns ou par les autres, ni surtout sur la "modularité de l'esprit" dont parle Jerry A. Fodor ${ }^{4}$ et que Dan Sperber a étendue depuis ${ }^{5}$, reconnaissons à ce type d'approche le mérite non négligeable d'exprimer, dans le vocabulaire de la théorie des systèmes, une propriété des langues trop peu étudiée.

Gustave Guillaume, interrogeant la définition saussurienne de la langue comme système, soulignait déjà que «chaque forme de langue doit être considérée comme l'expression de la commune relativité de ses emplois aux emplois des autres formes de la langue et l'ensemble des formes d'une langue comme un système de relativités réciproques» ${ }^{6}$. Il passait ainsi de cette idée de "système de relativités réciproques" à celle de la langue comme "système de systèmes" qui a

1 B. Combettes, "Questions de méthode et de contenu en linguistique du texte", Études de linguistique appliquée, $\mathrm{n}^{\circ}$ 87, Paris, Didier, 1992, p. 113.

2 M. Charolles, op. cit., p. 314.

3 H. NøLKE, Linguistique modulaire, Louvain-Paris, Éd. Peeters, 1994.

4 J. A. FODOR, The Modularity of Mind, Boston, M.I.T., 1983.

5 D. SPERBER, La contagion des idées, Paris, Éd. Odile Jacob, 1996 (chap. 6).

6 G. GuILlaUME, Temps et verbe, Paris, Champion, 1929, p. 124. 
amené Alain Berrendonner, au début de Principes de grammaire polylectale, à considérer la langue comme

un ensemble de sous-systèmes qu'il est légitime d'envisager synchroniquement et diachroniquement, comme autonomes. (...) Encore faut-il, après cela, montrer comment "tout se tient" quand même, c'est-à-dire rendre compte du fait que les sous-systèmes ne sont pas simplement juxtaposés, mais reliés ${ }^{1}$.

Dès 1981, un linguiste comme Robert de Beaugrande allait déjà dans la direction qui nous intéresse lorsqu'il notait que

Chaque sous-système d'un texte (par exemple le lexique, la grammaire, les concepts, les étapes d'un plan, les actes de parole, et ainsi de suite) fonctionne en partie d'après ses propres principes internes et en partie selon des demandes ou des rétroactions des autres sous-systèmes ${ }^{2}$.

Dans cette perspective, un texte peut être défini comme une configuration réglée par divers plans d'organisation ou sous-systèmes en constante interaction. Ces plans doivent être considérés comme autant de sous-théories ou "domaines"3 d'une théorie d'ensemble.

Au plan A, la presse écrite présente une utilisation spécifique de faits micro-linguistiques comme la nominalisation, les phrases passives, les phénomènes de reprise et de co-référence. Non seulement ces faits de langue méritent un examen attentif, mais ils doivent être mis en relations avec des faits macro-structuraux : système des temps dans le reportage sportif et dans les rubriques nécrologiques, phrases passives (en particulier dans les relations de catastrophes, les titres, les chapeaux, les brèves et les filets), discours relaté dans le genre que l'on pourrait appeler "relations de paroles" et dans le témoignage, anaphores démonstratives et fonctionnement du lexique dans les fragments de discours didactique si fréquents dans les articles de presse, etc.

1 A. Berrendonner et al., Principes de grammaire polylectale, Lyon, Presses universitaires de Lyon, 1983, p. 10.

2 R. de BEAugRANDE, "Critères d'évaluation des modèles du processus de lecture", in G. DENHIÈRE (éd.), Il était une fois..., Lille, Presses universitaires de Lille, 1984, p. 356.

3 B. COMBETTES, op. cit. 
STRUCTURE COMPOSITIONNELLE [plan C]

- STRUCTURE RHÉTORIQUE :

Plans de textes plus ou moins réglés par des genres discursifs.

- STRUCTURE SÉQUENTIELLE :

(Proto-)types de séquences de base : - Narratif

- Descriptif

- Argumentatif

- Explicatif

Modes d'articulation des séquences :

- Dialogal

- Suites linéaires :

- Séquences coordonnées

- Séquences alternées

- Insertion :

- Séquences enchâssées

Au plan C -qu'il s'agit toujours, je le rappelle, de mettre en rapport avec la généricité (inter)discursive-, on sera attentif, d'une part, aux plans de textes souvent assez contraints et qui organisent la dispositio de tel ou tel genre d'article : plan de texte canonique du reportage sportif ou du compte rendu/présentation de films, en particulier. C'est ce que j' appelle la "structure rhétorique" et l'on peut dire, à propos de la presse écrite, que cette structure rhétorique est plus ou moins réglée par les genres (inter)discursifs.

A ce niveau d'organisation, on sera attentif, d'autre part, à la complexité de la structuration séquentielle des genres à dominante argumentative, descriptive, narrative, explicative et dialogale. On sera attentif surtout aux modes (coordonnés, alternés ou enchâssés) d'articulation des séquences. C'est de cette façon que, rompant avec les typologies simplistes des textes, on entre dans la complexité de l'organisation compositionnelle. C'est très précisément sur ce point que j'ai besoin de rompre avec le principe du "tout narratif", dans le but d'essayer de mettre en évidence des différences, une hétérogénéité, une complexité compositionnelle autrement riches.

Les genres réputés narratifs, comme le fait divers et le reportage, ne présentent pas toujours une structure compositionnelle proprement narrative. Il semble également que, selon la visée (plan E d'organisation), la mise en intrigue narrative peut s'insinuer partiellement dans des éditoriaux, des billets d'humeur et ce que les journalistes appellent les "papiers d'idées" (pourtant majoritairement non narratifs dans leur organisation séquentielle). Notre modélisation de 
l'hétérogénéité compositionnelle des textes permet d'identifier soigneusement les modes d'insertion de ces moments narratifs dans les articles, leur fonction argumentative et l'espace (en termes quantitatifs) qui leur est réservé dans tel journal, tel type de "genre rédactionnel" et tel type de presse.

\section{STRUCTURATION PRAGMATIQUE}

- S. SÉMANTIQUE : contenu propositionnel (p)

Construction des objets du discours, «mondes», isotopies

DESCRIPTION

$=\ll$ DICTUM»

- E. ÉNONCIATION :

paramètres de l'acte d'énonciation et prise en charge des propositions où s'articulent $S(p) \& I$ (f) $=\ll$ MODUS»

- I. FORCE ILLOCUTOIRE : valeur d'acte du discours (f)

Buts, visée(s) du texte

ARGUMENTATION

La presse écrite illustre le fonctionnement des trois plans que je regroupe sous l'étiquette de "structuration pragmatique". Le plan sémantique (S) gère l'organisation du lexique et la construction d'un monde représenté qui peut être connu (allusions, usage des anaphores définies sans référent préalable) ou qui peut être nouveau (explicitations de type didactique). Le plan énonciatif (E) permet de travailler la question complexe de la présence de l'énonciateur dans son énoncé et donc de revenir linguistiquement sur la distinction proposée par tous les manuels de journalisme entre "information" (effacement du sujet de l'énonciation) et "commentaire" (présence assumée du sujet de l'énonciation). Enfin, le plan illocutoire (I) permet de travailler la conception journalistique des buts ou intentions communicatives aussi bien que les enchaînements de micro-actes de discours.

Afin d'illustrer certains de ces plans en restant dans le sujet narratologique qui nous retient, examinons les trois catégories que Genette distingue: le récit, l'histoire et la narration. En fait, ces trois catégories, constitutives du fait narratif dans sa globalité, correspondent à trois des plans d'organisation de tous les textes.

- Le récit ou texte-racontant correspond aux plans $\mathrm{T}$ et $\mathrm{C}$ d'organisation, c'est-à-dire au texte proprement dit, comme suite de proposi- 
tions, à la continuité textuelle. Le concept de "récit" n'est donc qu'une théorisation sectorielle de la continuité textuelle narrative qu'il faut, selon moi, comparer aux autres sortes de continuités : descriptive, argumentative, explicative et dialogale.

- L' histoire ou contenu raconté correspond au monde du texte, c'està-dire au plan sémantique, ce que l'on appellerait utilement l'univers diégétique du texte narratif. Les personnages et leurs actions, le temps représenté et l'espace, sont les constituants majeurs du Monde du texte. Il n'y a pas de texte sans représentation discursive d'objets du discours. Le concept d' "histoire" doit donc, lui aussi, être considéré comme une théorie sectorielle de la structuration sémantique d'un type particulier de structure compositionnelle. Notons que c'est à ce niveau sémantique que se pose la question de la fictionalité. Ajoutons que c'est également à ce niveau sémantique qu'on examinera les classifications en grandes "familles événementielles": nouvelles politiques, catastrophes (naturelles, écologiques, accidents d'avions), voyages/visites (présidentiels, ministériels, royaux, princiers, papaux), rencontres d'hommes politiques, conflits armés, conflits sociaux, décès de personnalités, manifestations, etc.

- La narration, enfin, ou raconter comme acte, correspond avant tout au plan énonciatif (E) qu'il s'agit d'étudier avec les faits d'emboîtements possibles de la narration dans les témoignages, avec les paroles rapportées en général (discours direct, indirect ou "narrativisé"), avec les faits de polyphonie (ironie, négation, concession), l'utilisation des temps verbaux (du conditionnel journalistique au passé simple), etc. Le concept de "narration" est donc une théorisation sectorielle de l'énonciation en régime narratif.

Au plan illocutoire (I) se pose la question des buts ou visées et c'est par lui qu'on rejoint la dimension discursive, en retrouvant la question des intentions communicatives. On peut aborder la fiction en termes sémantiques (plan S), mais la fiction est également déterminée par le plan I d'organisation. A ce niveau, la fiction est un contrat fictionnel ou non-fictionnel que le lecteur passe avec le texte. Un troisième niveau de fictionalité est micro-textuel (plan $T$ ) : rôle des phrases hypothétiques à l'imparfait, du conditionnel, des opérateurs de type "il était une fois...". Au plan I, on tiendra compte des classifications des rubriques de la presse écrite sur la base des buts/intentions communicatives poursuivis, depuis le classement en deux grandes catégories: informer (sans prendre parti) et commenter (donner un avis personnel) jusqu'aux classements un peu plus fins, 
mais qui demandent à être linguistiquement clarifiés : narrer ce qui s'est passé (faits politiques, faits divers...), exprimer une opinion (critique, lettres de lecteurs, interviews...), analyser (textes émanant généralement d'experts), décrire (portraits de personnalités, descriptions de sites, d'objets), proposer/demander un service (petites annonces, offres d'emploi), vendre (publicités), divertir (mots croisés, dessins humoristiques, bandes dessinées, feuilletons...).

A titre d'exemple, examinons très rapidement deux "Nouvelles en trois lignes" rédigées, en 1906, par Félix Fénéon pour le grand quotidien Le Matin :

\section{T1}

Ayant terrassé l'afficheur Achille, ils le tirèrent sur toute la longueur de la passerelle d'Alfort-ville, puis le précipitèrent.

- Plan T : La texture micro-linguistique de cette phrase complexe est intéressante. Le texte s'ouvre sur une structure syllabique proche du rythme d'un décasyllabe (Ayant terrassé [5] l'afficheur Achille [5]) dont le second hémistiche est particulièrement allitérant: $[\mathrm{a}+\mathrm{ich}]^{1}$ puis $[a+c h i]$. Cette structuration de type poétique ne se retrouve plus qu'à la fin : [PuIs le PRécIPItèRent]. A un niveau micro-linguistique, on relèvera surtout un phénomène de reprise anaphorique pronominale classique (Achille $>$ LE (puis) LE) et un type tout à fait agrammatical : le pronom personnel ILS ne comporte pas de référent. Il est évident que cette agrammaticalité fait pleinement sens au plan (C) sémantique : les agresseurs non identifiés restent très légitimement dans un anonymat que l'enquête devra lever.

- Plan C : La structure de ce petit texte est-elle narrative ? Je réserve la réponse à cette question pour la deuxième partie de ce travail.

- Plan S : L'information sur le monde diégétique est donnée en quelques mots. Il est répondu classiquement aux questions QUI? (nom propre et fonction de la victime et anonymat des agresseurs), QUOI? (agression détaillée en une chaîne d'actions : terrasser, tirer puis précipiter), OŨ? (Passerelle d'Alfort-ville). Seules les questions QUAND ? (après tout partiellement résolue par la date du journal : la veille?) et surtout POURQUOI? restent dans l'ombre. Cette question essentielle de la motivation actionnelle (intentions de tuer certes mise 1 Je transcris graphiquement ce qui est phonique, pour la lisibilité par des non-
spécialistes. 
en évidence, mais pour quel motifs, avec quelles raisons d'agir de la sorte ?).

- Plan E : La structure énonciative de ce petit texte est particulièrement distanciée. L'énonciateur semble absent de son énoncé. En fait, conformément à l'esthétique même de Félix Fénéon, je me demande si cette absence énonciative ne s'explique pas par un choix rhétorique que je vais expliciter avec le dernier plan d'organisation.

- Plan I : Les énoncés successifs sont tous de type assertif : ils disent que quelque chose est. L'intention illocutoire qui s'en dégage semble être -conformément à ce qu'on vient de dire de la distanciation énonciative manifeste-d'informer objectivement. Mais l'écrivain pointe son nez sous le travail du style dont j'ai parlé plus haut (plan T). On bascule ainsi dans le genre épidictique (démonstratif des latins) de la rhétorique antique, ce genre qui laisse admirer moins le contenu que la façon dont ce qui est dit est formulé : l'art de l'énonciateur.

$\mathrm{T} 2$

Dans le lac d'Annecy, trois jeunes gens nageaient. L'un, Janinetti, disparut. Plongeon des autres. Ils le ramenèrent, mais mort.

- Plan T : La texture micro-linguistique de T2 diffère de celle de T1. Nous avons, cette fois, quatre phrases typographiques, dont une nominale. La texture rythmique est particulièrement intéressante: le texte s'ouvre, en effet, sur un alexandrin : "Dans le lac d'Annecy [6], trois jeunes gens nageaient [6]". Celui-ci marque de son équilibre l'équilibre euphorique de la situation initiale du récit. Le tissage anaphorique est très rigoureux : un groupe "trois jeunes gens" est constitué et scindé ensuite en deux: "I'un", la victime, acquiert (comme dans T1) un nom propre : "Janinetti", avant d'être repris par le pronom LE. "Les autres" restent anonymes : «LS».

- Plan C : La structure narrative de ce petit texte sera examinée plus loin.

- Plan S : L'information sur le monde diégétique est donnée en quelques mots. Il est répondu classiquement aux questions QUI ? (je viens d'en parler), QUOI ? (accident suivi d'une chaîne d'actions, OÙ ? (le Lac d'Annecy). La question du temps reste comparable à celle de T1. La question POURQUOI ? qui porte sur la motivation actionnelle des actions/réactions est implicitement donnée : intention de sauver. 
- Plan E : La distanciation énonciative est, ici aussi, grande et soulignée également par le passé simple.

- Plan I : Les remarques formulées à propos de T1 sont ici confirmées : comment ne pas admirer le style de ce petit texte et donc l'art de son auteur? La rupture du rythme de l'alexandrin par le drame, la phrase nominale centrale et surtout la chute finale introduite par le connecteur "mais", sont autant de traces d'une écriture au service étroit d'effets de sens concentrés à l'extrême.

\section{Une théorie pragmatique et textuelle du récit : pour une approche en termes de gradients de narrativité}

Cette "définition restreinte du récit" s'inscrit dans une théorie qui rompt radicalement avec le "tout narratif" de la sémiotique, "tout narratif" qui semble parcourir certains travaux de l'O.R.M. Je ne cite ici qu'un ouvrage dont la visée didactique explique probablement le choix d'une définition large qui ne tient pas compte des degrés de narrativité dont je vais parler.

Dans le journal, il n'y a pas que l'histoire fictive du feuilleton à être rédigée comme un récit, en suivant les normes d'une certaine dramaturgie. Dans chaque numéro d'un organe de presse, tout -ou presque- répond aux mêmes lois narratives. De la météo aux nécrologies en passant par les petites annonces et, évidemment, l'ensemble du "rédactionnel" classique. (...) L'écriture de presse a donc une base bien simple: arriver à adopter le style du récit, de la narration ou, si l'on préfère, de la relation de l'événement ${ }^{1}$.

Si nous pouvons être d'accord sur le fait que tout peut être narrativisé, raconté et que le récit est une forme textuelle particulièrement bien reçue par les lecteurs, je ne comprends pas que dans le guide d'écriture de la page 38 , à propos des procédures à suivre pour la rédaction d'un article, on trouve, entre "(2) Mesurer ses ardeurs" et "(4) Mettre en œuvre les spécificités de l'écriture communication-

1 Fr. AntoIne et al., Écrire au quotidien. Pratiques du journalisme, Bruxelles, Éd. Vie ouvrière, 1996 (2e éd.), p. 19. Cet ouvrage, qui ne correspond qu'indirectement aux options théoriques de l'ORM, a été choisi comme simple exemple d'une direction générale très éloignée de la mienne. 
nelle", un point (3) : "Établir une structure narrative". Il y a là une confusion évidente entre la planification d'un texte (les anciens disaient sa dispositio) et le cas très particulier du choix d'une organisation narrative. Celle-ci est d'ailleurs détaillée dans le manuel, en trois points qui n'ont rien de proprement narratif :

- du général au particulier ou l'inverse?

- donner d'emblée toute l'information puis la développer ou la dévoiler progressivement?

- respecter la chronologie des faits et des propos ou faire un "montage" personnel?

On voit bien là qu'on parle de la distribution du référent ou objet du discours tout au long de la continuité textuelle (entre le début et la fin de l'article). Le problème d'écriture à résoudre est celui de la mise en texte d'une information relative à un événement ou à un contenu quelconque.

La question intéressante à débattre est la suivante : que gagne-ton à dire qu'une petite annonce ou la rubrique météo sont des récits? A mon avis, le déficit est double :

- déficit du côté de la textualité

- déficit du côté du récit lui-même.

\subsection{Un déficit dans l'approche de la textualité}

A mon sens, les meilleures études du genre des «petites annonces» parlent de descriptions argumentatives ${ }^{1}$ voire même de dialogue $^{2}$. Si on ne parle pas de description, il semble plus pertinent de considérer une petite annonce comme un dialogue en quête que comme un récit. En effet, la petite annonce est certainement plus proche d'une catégorie dérivée du dialogue comme la lettre que de la narration.

Il me semble que la catégorisation des petites annonces dans les descriptions argumentativement très marquées est techniquement la plus précise. Toutefois, ceci n'exclut pas qu'une petite annonce puisse

1 J.-M. Privat, "Les petites annonces matrimoniales ou la rhétorique des descriptions argumentatives", Pratiques, ${ }^{\circ}$ 56, Metz, 1987, p. 101-119.

2 M. BоnномmE, "Le dialogue en quête dans les petites annonces", in Le dialogue, Colloque d'Albi, Toulouse, Presses de l'Université de Toulouse-le-Mirail, 1990, p. 147-166. 
prendre -exceptionnellement, très partiellement et de façon parodique- la forme d'un récit. Ainsi cette petite annonce du Nouvel Observateur que cite Marc Bonhomme ${ }^{1}$ :

Un gentilhomme habitant une île paradisiaque et Paris parcourait en solitaire sa 39 ème année. Un jour, à la recherche du royaume des cœurs purs, il rencontra une très belle princesse. Ensemble ils vécurent et ils connurent tendresse, fidélité, humour, bonheur et amour. Adressez-moi une photo. Un jour, peut-être, vous conterez notre histoire. Ecrire journal, réf.432/7 $\mathrm{R}$.

Il est, selon moi, beaucoup plus productif d'expliquer comment des genres non narratifs peuvent recourir au récit en enchâssant des moments (séquences) narratifs dans leurs développements argumentatifs ou explicatifs. Quand la rhétorique de la dispositio judiciaire codifie la place de la narratio dans le plaidoyer, elle apprend vraiment à écrire et à composer. C'est bien d'une rhétorique généralisée de la composition dont nous avons besoin.

Je ne prendrai qu'un deuxième exemple de déficit en termes de textualité. Je le trouve à la page 78 d'Écrire au quotidien:

Au type classique de l'interview sur le mode purement interrogatoire (question-réponse en alternance) émerge un genre beaucoup plus souple et plus naturel (mais plus "travaillé" aussi malgré les apparences) : l'interview-conversation. A un troisième stade, l'interview-récit, le journaliste pousse son intervention personnelle jusqu'à reprendre complètement en main la narration et à ne plus faire parler en style direct son interlocuteur qu'au gré de son humeur, les autres propos étant résumés ou explicités par lui ${ }^{2}$.

Cette fois, la confusion me paraît être entre la construction dialogale et la construction monologale. Cette dernière semble assimilée abusivement avec la narration. Le problème énonciatif du discours relaté direct, indirect ou narrativisé, permettant de résumer les propos de quelqu'un, est une question de mise en forme énonciative (plan D d'organisation) et pas du tout une question de mise en récit (plan B). Je passe sur la distinction technique entre dialogue et conversation...

1 Ibid., p. 158.

2 Fr. ANTOINE et al., op. cit., p. 78. Les auteurs persistent page 79, à propos du choix entre le tutoiement et le vouvoiement, en parlant d'“authenticité du récit qui n'a pas à altérer [la] connivence [entre interviewer et interviewé]". 
les travaux de ces dernières années les opposant comme l'écrit (dialogal) et l'oral (conversationnel).

\subsection{Déficit dans la définition du récit et dans l'approche de la complexité de l'opération de mise en intrigue}

Il me semble que le récit est certes une façon, pour l'homme, de penser le temps : moyen de donner sens au temps et ainsi de dire le monde comme le dirait Paul Ricœur. Mais il me semble que le récit est surtout un mode de réflexion et d'expérimentation qui porte plus globalement sur l'agir humain (dont le temps n'est qu'un des cadres ou paramètres). L'opération de mise en intrigue, qui chez Ricœur luimême définit le récit, gagne à être affinée linguistiquement en affirmant d'abord que le couple nœud \& dénouement constitue l'élément déterminant de toute mise en intrigue.

Il est nécessaire de compléter en ce sens la définition partiellement juste que rappellent M. Mouillaud et J.-F. Tétu :

Le récit est un ensemble clos (...), c'est une marque essentielle de sa structure. On sait aussi que la fonction du récit est d'organiser, entre la situation par laquelle il s'ouvre et celle qui l'achève, une procédure d'inversion ou de transformation des contenus. C'est précisément en cela que le récit est fondamentalement différent des actions, ou des suites d'actions de la vie quotidienne, différent de la réalité dont n'importe quel lecteur a l'expérience. Car le monde, la réalité -et il n'est pas un homme qui ne le sache, et ne le vive, dans l'expérience même qu'il a de sa vie et du monde-n'a pas de début ni de fin; ou, si cette affirmation paraît excessive, le reél ne possède pas de début ou de fin qui soient repérables de l'extérieur et de façon assurée ${ }^{1}$.

La question de la transformation des prédicats de la situation initiale $-d$ 'ailleurs facultative et autrement complexe ${ }^{2}-$ n'atteint que les bornes de la construction. Parler de "transformation" (éventuelle) ne permet pas de dire les modalités de cette opération complexe. Or

1 M. Moulllaud et J.-Fr. TÉtu, op. cit., p. 173.

2 Voir à ce propos J.-M. ADAM et Fr. REVAZ, L'analyse des récits, Paris, Éd. du Seuil, coll. Mémo $\mathrm{n}^{\circ} 22$, chapitre 12. 
c'est précisément là que la mise en intrigue prend place et tout son sens.

Si nous revenons sur la structure compositionnelle des deux "Nouvelles en trois lignes" de Félix Fénéon, nous constatons que ces deux faits divers présentent au moins une différence importante.

T1 relate linéairement trois actions successives : terrasser l'afficheur, le tirer sur la passerelle, le précipiter en bas. Dans cette séquence actionnelle, aucun des faits ne se distingue des autres au point de pouvoir être considéré comme un nœud et/ou comme un dénouement. Les trois actions s'enchaînent et elles composent une macro-action agression brutale ou meurtre.

En revanche, dans T2, un événement particulier -la disparition de Janinetti- vient perturber un équilibre initial que le texte prend soin de poser. Cet événement, qui contraste fortement avec ce qui précède, forme ce qu'on peut sans hésiter appeler le nœud de l'intrigue. La réaction des compagnons de Janinetti -le plongeon- constitue une tentative de rétablir l'équilibre initial. Le dénouement-ramener celui qui a disparu- laisse d'abord envisager la résolution du drame, mais il s'avère, au bout du compte, déceptif. La situation finale est définitivement négative: Janinetti est mort. Il y a donc bien eu transformation et, de plus, résolution déceptive.

La structure compositionnelle de T2 est la suivante :

\begin{tabular}{|c|c|}
\hline $\begin{array}{l}1 \text { - Situation initiale } \\
\text { à l'imparfait /VIE/ \& /EUPHORIE/ }\end{array}$ & $\begin{array}{l}\text { Dans le lac d'Annecy, } \\
\text { trois jeunes gens nageaient. }\end{array}$ \\
\hline $\begin{array}{l}2 \text { - Nœud (= déclencheur) } \\
\text { souligné par le passé simple }\end{array}$ & L'un, Janinetti, disparut. \\
\hline $\begin{array}{l}3 \text { - Action (= réaction) } \\
\text { dans une simple phrase nominale }\end{array}$ & Plongeon des autres. \\
\hline 4 - Dénouement & Ils le ramenèrent, \\
\hline $\begin{array}{l}5 \text { - Situation finale } \\
\text { qui inverse brutalement des prédicats } \\
\text { implicites de la situation initiale : } \\
\text { /MORT/ \& /DYSPHORIE/ }\end{array}$ & mais mort. \\
\hline
\end{tabular}

Le découpage de ce texte de quatre phrases laisse apparaître une structure en cinq propositions qui constitue la séquence narrative minimale prototypique : 


\section{Séquence narrative}

Action ou

Évaluation

Pn 3

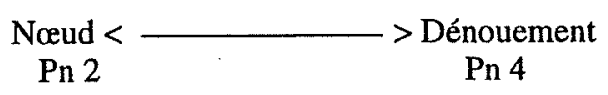

Situation

Situation

initiale $<$ $>$ finale

Pn 1

Pn 5

phrase 1 phrase 2 phrase $3 \quad$ phrase 4

Dans T2, chaque proposition correspond à un constituant différent de la structure canonique. Dans une séquence narrative plus longue, il serait nécessaire d'opérer des regroupements de propositions en macro-propositions narratives, notées "Pn" dans le schéma.

Cette schématisation en triangle souligne la hiérarchisation des propositions ainsi que les rapports de correspondance : la Situation finale (Pn5) renvoie à la Situation initiale (Pn1) et le Dénouement (Pn4) est symétrique au Nœud (Pn2). L'Action (ou Évaluation, si au lieu d'agir le narrateur ou un personnage évalue seulement la situation) (Pn3) est au centre du dispositif, elle résulte directement du Nœud (Pn2).

On peut observer des degrés divers d'actualisation des composants de ce schéma canonique. Si le Dénouement est clairement exprimé, la Situation finale n'a pas forcément besoin d'être explicitée. A l'inverse, l'impasse peut être faite très exceptionnellement sur le Dénouement, à condition que la Situation finale soit précisée. En revanche, le Nœud, déclencheur de l'action, présente un caractère obligatoire.

On parlera de mise en forme narrative lorsque des propositions seront interprétables comme nœud-Pn2 et comme dénouement-Pn4. 
En d'autres termes, bien que ces deux textes ne présentent que des faits bruts, sans la moindre explicitation des raisons d'agir des acteurs, en tenant compte de cette définition très stricte du récit, $\mathrm{T} 2$ présente un plus fort gradient de narrativité que T1 qui ressemble plutôt à une simple description d'actions. T1, du fait même de son incomplétude, semble n'être qu'un fragment (noyau actionnel Pn3, par exemple) de récit. En effet, la première action est donnée sans que soit établie la moindre situation initiale et la dernière action s'achève très mystérieusement : où le corps de l'afficheur Achille a-t-il été précipité? Au lecteur de rétablir qu'éventuellement la passerelle renvoie à l'eau et donc que le corps a pu être jeté à l'eau.

Pour expliciter et surtout essayer d'approfondir la portée de cette distinction entre le plus haut degré de narrativité du second fait divers de Fénéon par rapport au plus faible degré de narrativité du premier, considérons deux exemples journalistiques plus développés, relatifs à une catastrophe naturelle survenue le 24 septembre 1993, dans le Valais Suisse. Au terme de leur analyse, nous essaierons de classer les quatre textes sur une échelle qui rende compte du fait qu'un jugement spontané classerait certainement les quatre textes dans la classe des récits, alors qu'une étude plus technique repère des distinctions, à mon sens, importantes.

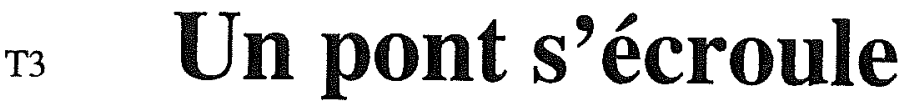 \\ Lavey (VD) : l'ouvrage métallique de 200 tonnes n'a pas résisté au Rhône}

Face au Rhône déchaîné, le vieux pont de Lavey, dans le Chablais vaudois, n'a pas résisté longtemps : il est tombé en un temps record, hier matin à $1 \mathrm{~h} 40$.

Daniel Prada, sapeur-pompier à Lavey, a assisté, impuissant, à la chute de cet édifice métallique de 200 tonnes. «J'étais sur place depuis 20 heures pour bloquer la circulation à l'entrée du pont. Vers minuit, le niveau du Rhône atteignait près de cinq mètres (N.d.l.r. : au lieu des 50 centimètres habituels) et la vitesse du courant avoisinait 70 $\mathrm{km} / \mathrm{h}$. Tout à coup, plusieurs centaines de mètres cubes de troncs se sont pris dans l'armature métallique du pont : cela a fait un effet barrage et le pont s'est écroulé.»

En tombant, l'ouvrage a arraché des lignes et de nombreux habitants de Lavey ont été privés de communication téléphonique durant la journée. Craignant de semblables accidents, les pompiers ont, de leur côté, interdit la circulation sur les toutes proches passerelles de Massongex et d'Illarsaz.

- L. K.

(Le Matin, dimanche 26 sept. 1993) 
L'analyse du témoignage du pompier permet de distinguer une mise en récit proprement dite d'une simple relation de faits. A première vue, il semble que l'on ait structurellement affaire ici à un récit canonique (soit un certain degré de narrativité) :

\begin{tabular}{|l|l|}
\hline $\begin{array}{l}\text { - Situation initiale : } \\
\text { Description des faits } \\
\text { dominée par l'imparfait. }\end{array}$ & J'étais sur place... avoisinait $70 \mathrm{~km} / \mathrm{h}$. \\
- Nœud [?] : & $\begin{array}{l}\text { TOUT A COUP... se sont pris dans } \\
\text { l'armature métallique du pont. } \\
\text { Cela a fait un effet (de) barrage. } \\
\text { - Évaluation (explicative) : } \\
\text { - Dénouement [?] : }\end{array}$ \\
$\begin{array}{l}\text { - Situation finale : } \\
\text { déplacée de sa place canonique s'est écroulé. } \\
\text { en fin de témoignage, dans le } \\
\text { dernier paragraphe du texte } \\
\text { (conséquences du désastre) et } \\
\text { dans la photo située sous le } \\
\text { texte, photo qui ne montre plus } \\
\text { que le vide de la chaussée } \\
\text { (pont disparu). }\end{array}$ \\
\hline
\end{tabular}

Il manque, pour qu'une réelle mise en intrigue narrative se développe et qu'un haut degré de narrativité soit atteint, deux ingrédients essentiels. D'une part, l'action relatée a beau émaner d'un témoin humain, les faits ne portent pas la moindre trace d'intentionnalité d'un actant humain (ou au moins anthropomorphe, humanisé) : personne ne tente une action de résistance, les hommes ne sont que témoins sur la berge du combat que se livrent la nature et le pont. D'autre part, les bifurcations potentielles des possibles narratifs n' apparaissent pas une seconde. A partir de "Tout à coup", au lieu d'un développement narratif, on trouve seulement une chaîne événementielle qui progresse de causes en effets. Cet organisateur temporel introduit d'ailleurs un événement marqué grammaticalement dans sa causalité matérielle ("se sont pris"). La causalité pure est renforcée par l'explicatif "cela a fait un effet (de) barrage". Un "et" relie ainsi (succession temporelle et causale) la cause événementielle à l'effet. Enfin, la "situation finale" n'est que l'indication d'un résultat. "Tout à coup" donne superficiellement l'allure d'un récit à ce qui n'est, en fait, qu'une 
relation de faits, une relation d'événements (même pas d'actions). Je dirai donc que T3 possède un faible degré de narrativité. Sur une échelle, il serait situé entre T1 (plus bas degré de narrativité) et T2.

A titre de comparaison, voyons comment une intentionnalité et une mise en intrigue peuvent structurer un énoncé en lui conférant, cette fois, un très haut degré de narrativité.

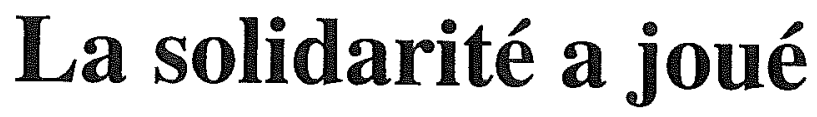

Les habitants de Brigue ont traversé des moments dramatiques. (...)

Un pompier qui voulait porter secours aux habitants d'un immeuble situé en face de la gare s'est retrouvé pris dans la boue comme dans des sables mouvants. Il allait s'enfoncer inexorablement et mourir étouffé. Heureusement, il se trouvait à la verti- cale de l'immeuble de $M$. Siegfried Escher, recteur du collège de Brigue. $M$. Escher est guide de montagne. Il réussit à lancer l'une de ses cordes au pompier en difficulté. $M$. Escher et ses voisins le tirèrent de [la] masse gluante. (...)

(Le Nouvelliste, lundi 27 septembre 1993)

Ce petit récit développe, en le dramatisant, un épisode que l'on pourrait appeler : épisode du sauveteur secouru. Les acteurs ont certes une identité inégale (le pompier ne jouit pas de l'identification descriptive assez développée du sauveteur : $M$. Escher), mais ce déséquilibre descriptif permet, en fait, de constituer $M$. Escher en héros d'un épisode dans lequel il faut bien distinguer un sujet d'état (le pompier) d'un sujet de faire (le sauveteur M. Escher), agent de la transformation narrative du sujet d'état menacé d'abord par l'engloutissement puis sauvé. Le changement de temps des verbes souligne cette différence : le pompier est gratifié d'imparfaits et seulement d'un passé composé qui tranche avec les passés simples qui ponctuent l'action hérö̈que finale de $\mathrm{M}$. Escher et des voisins. On peut, cette fois sans hésitation, détailler la structure de cette mise en intrigue. On a bien une succession de macro-propositions narratives $(\mathrm{Pn})$ qui constituent les étapes d'une très canonique mise en récit (cf. tableau page suivante).

Avec l'introduction d'une évaluation-morale en titre, le degré d'élaboration de la signification s'élève très nettement et l'on se trouve confronté formellement à une propriété majeure de tout récit : être écrit à partir de sa fin. La dimension configurationnelle, dont parle Paul Ricœur, est ici explicitée et nous sommes au plus près de la fable morale. 
- Pn1-Situation initiale:

Description des faits dominée par l'imparfait.

\section{- Pn2-Neud:}

Dramatisation accentuée par une comparaison («comme dans des sables mouvants») qui rattache l'événement à des scénarios d'engloutissements présents dans l'imaginaire des lecteurs.

- Pn3-Évaluation-explication: Évaluation de la situation tragique créée par le nœud, cette phrase n'utilise pas pour rien le futur proche: elle présente ce qui risque d'advenir, elle place le lecteur en une bifurcation des possibles narratifs qui ouvre sur une mort possible imminente.

\section{- Pn4-Dénouement:}

Issue positive liée à l'action des sauveteurs, cette macroproposition est ouverte par l'adverbe "heureusement" qui souligne d'entrée le soulagem voisins le

Le sauveteur, M. Escher, est tout entier dans cette partie résolutive du récit.

- Pn5-Situation finale:

ellipse de cette situation finale qui inverse Pn1: le sauveteur en péril est sauvé.

\section{- Pn $\Omega$-Évaluation finale} ou "Morale":

Le titre de l'article donne à relire l'épisode comme un modèle des réactions positives de la population de la ville de Brigue à la catastrophe naturelle.
Un pompier qui voulait porter secours aux habitants d'un immeuble situé en face de la gare.

s'est retrouvé pris dans la boue comme dans des sables mouvants.

Il allait s'enfoncer inexorablement et mourir étouffé.

Heureusement, il se trouvait à la verticale de l'immeuble de M. Siegfried Escher, recteur du collège de Brigue. M. Escher est guide de montagne. Il réussit à lancer l'une de ses cordes au pompier en ment. difficulté. $M$. Escher et ses tirèrent de [la] masse gluante.

La solidarité a joué. 


\section{Pour conclure}

On voit ainsi comment l'écriture transforme un épisode d'une catastrophe naturelle en fait digne d'être relaté ou raconté. Le combat de titans du vieux pont contre les flots déchaînés participe à une métamorphose dramatisante du réel, il prépare son interprétation symbolique. Mais, dans le cas de T3, le choix de la relation plutôt que de la mise en récit complète permet de mettre l'accent sur le cours inexorable des événements. Tout au contraire, dans l'épisode de l'engloutissement évité (T4), la mise en intrigue permet, cette fois, d'insister sur la façon dont le cours tout aussi inexorable des choses se trouve modifié par les actions héroïques et solidaires des actants humains. L'écho avec le texte T2 du sauvetage manqué dans le lac d'Annecy est ici patent. Les deux textes présentent un même renversement sémantique (certes sémantiquement inverse), mais T4 se distingue de T2 par l'évaluation-morale explicite. L'interrogation de l'agir humain étant plus développée, je dirai que T4 possède un plus haut degré de narrativité que $\mathrm{T} 2$.

On obtient donc, en fonction de ces critères de définition du récit (je renvoie également au chapitre 5 du Texte narratif ${ }^{1}$ pour un exposé complémentaire sur ces critères), une échelle qui permet de proposer un classement des textes étudiés ci-dessus. Ce classement sur un continuum exclut moins absolument $\mathrm{T} 1$ et $\mathrm{T} 3$ de la classe des récits qu'il ne tente de tenir compte du jugement spontané qui les considérerait certainement comme des récits. Les définitions techniques proposées tentent sinon de corriger radicalement le jugement spontané, du moins de le nuancer et de l'affiner en sortant d'un "tout narratif" qui, selon moi, masque trop les différences :

\begin{tabular}{|c|c|c|c|}
\hline \multicolumn{4}{|c|}{ NARRATIVITÉ } \\
\hline $1-1$ & $\begin{array}{l}\text { Fail } \\
\text { (des }\end{array}$ & $\begin{array}{l}\text { egré } \\
\text { ions d'actions) }\end{array}$ & $\begin{array}{l}\text { Haut degré } \\
\text { (mise en intrigue et } \\
\text { interrogation de l'agir humain) }\end{array}$ \\
\hline & T1 & T3 & T2 \\
\hline
\end{tabular}

1 J.-M. ADAM, Le texte narratif, op. cit. 